\title{
Design of Wireless Electronic Price Tag Based on STM32L Microcontroller
}

\author{
Yingzhi Wang ${ }^{1, a}$, Tailin Han $^{1, b}$, Jia Yang ${ }^{2, c}$ \\ ${ }^{1}$ Changchun University of Science and Technology, Changchun 130022, China \\ ${ }^{2}$ Jilin Jianzhu University, Changchun 130118, China \\ a22473698@qq.com, bhantl@cust.edu.cn, '109188016@qq.com
}

Keywords: Wireless Electronic Price Tag, Electronic Paper Displays, STM32L Microcontroller.

\begin{abstract}
Wireless electronic price tag is designed with the Electronic Paper Displays' low power consumption, using STM32L152RD single-chip microcomputer as the core. Practice has proved that electronic price tag of low cost and power consumption can update the commodity information on the remote wireless price tag.
\end{abstract}

\section{Introduction}

With the vigorous development of the retail industry, the supermarket scale is larger and larger. Also, more and more kinds of goods are in the supermarket. All types of sales promotion result in commodity prices changing more and more frequently. It leads plenty of time and energy to be consumed on the replacement and management of price tag and inevitably, which will emerge mistakes. It is for these reasons that we advance the wireless electronic price tag invention. Electronic price tag is an electronic display device placed on the shelves to alternate the traditional paper price tag.

Each electronic price tag is connected to the mall computer database via wireless communication technology, and the latest commodity price information displays through Electronic Paper Displays (EPD), completing updating information of product .Electronic price tag possesses various advantages, such as real-time updating information, reducing labor intensity, avoiding errors and so on.

\section{The system hardware design}

The design of wireless electronic price tag mainly completes commodity prices and other information displayed, and the information on the label can be remotely modified. System principle diagram is shown in Figure 1.

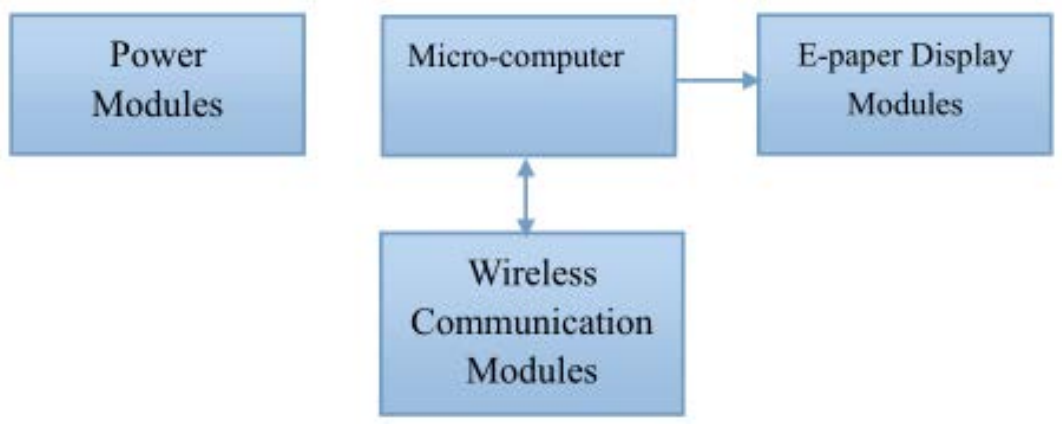

Figure 1 : System principle diagram

Single-chip microcomputer selection. STMicroelectronics ARM Cortex ${ }^{\mathrm{TM}}$ - M3 core 32bit STM32L152C8 microcontroller is selected to be responsible for the overall operation of the system. Its features are: operating frequency is $32 \mathrm{MHz}$, it is divided into 6 kinds of operating modes, it can complete the task with the lowest power consumption at any set time, and it is integrated USART 
interface, USB2.0 full-speed interface etc. SPI communication mode can be used to communicate with external devices. The debug mode is in the SWD and JATG interface.

Power Module Design. CR2032 button battery power systems. The 3V button battery provides power for the MCU STM32L152C8, wireless communication module and receiving e-paper display modules. Specific power supply circuit diagram is shown in Figure 2, where the label VCC provides power for microcomputer and wireless communication module and the label VCCEPD provides power for EPD.

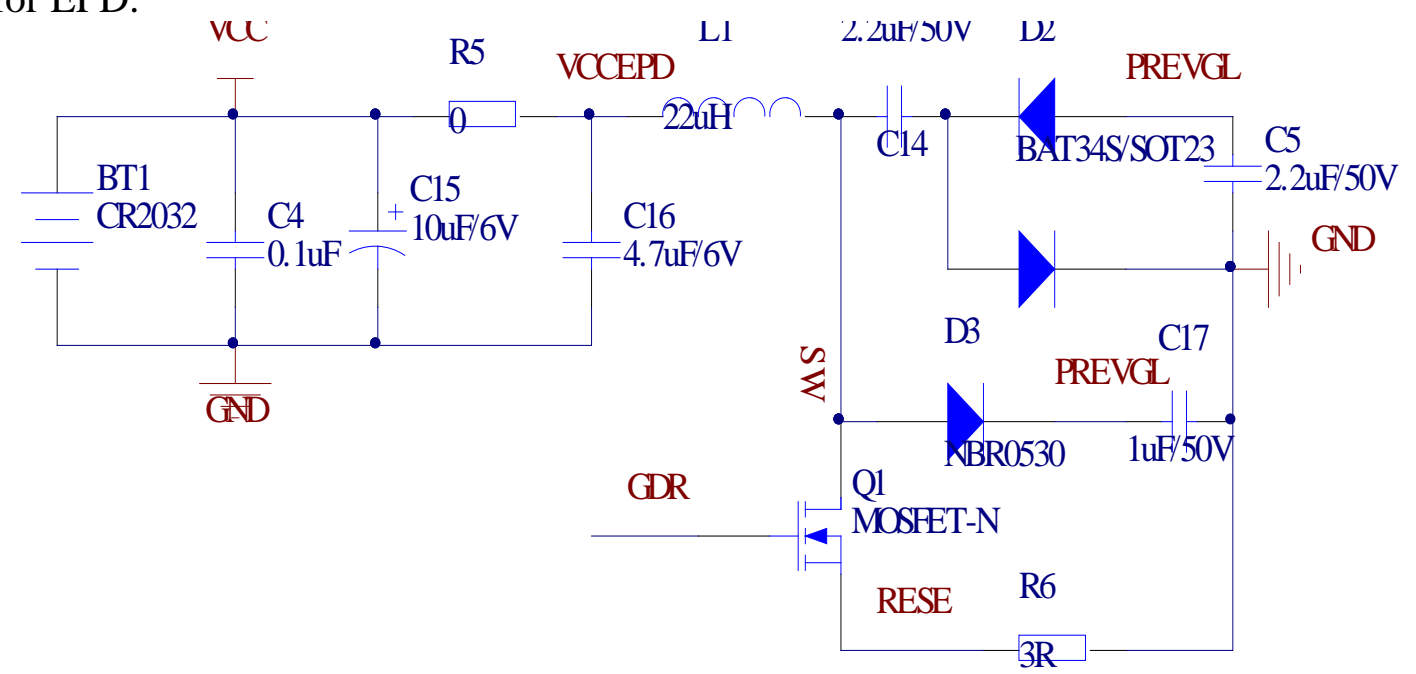

Figure 2: System power module circuit diagram

Wireless Communication Module. Wireless communication module chose TI's CC1100 chip. It has low power consumption, high receiver sensitivity, programmable speed and optional operating frequency features. Except external circuit of CC1100, its microcontroller interface is shown in Figure 3.

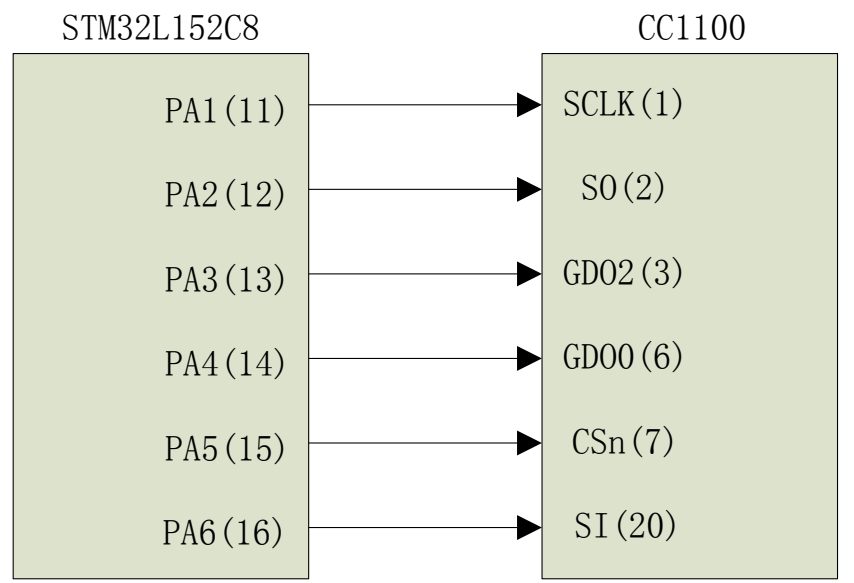

Figure 3: Microcontroller and CC1100 Interface Figure

EPD module interface design. Dalian Jin Xian Co.Ltd. 2.1-inch EPD is selected, which uses E-LNK technology. It is characterized by: (1) it can be read in sunlight; (2) different from liquid crystal, Panel can be thin and light using ink; (3) 180-degree viewing angle, the same effect as plain paper; (4) high-resolution, $172 \times 72$ pixels; (5) depended on a voltage pulse to refresh, the actual power consumption is zero when refreshment is not need. SPI bus control mode use SCLK, SDIN, CS three lines to control data display. The displayed data includes commodity prices, place of origin, date of production and so on. EPD and microcontroller interface circuit are shown in Figure 4. 


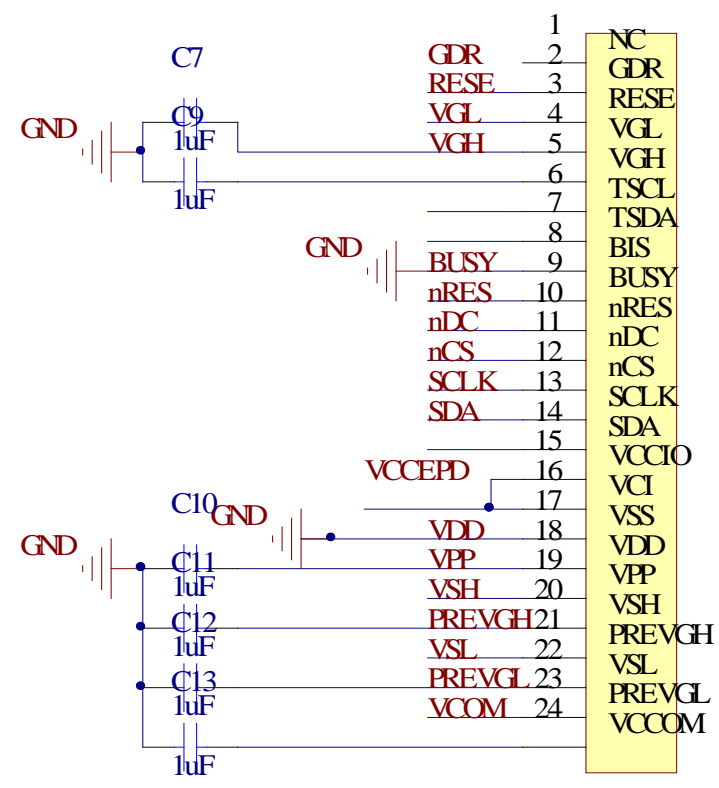

Figure 4: EPD and microcontroller interface circuit

\section{System Software Design}

Program is developed under the company Keil uVision4 development environment. Subroutines include: wireless communication module initialization, the EPD module initialization. The main program flow chart is shown in Figure 5.

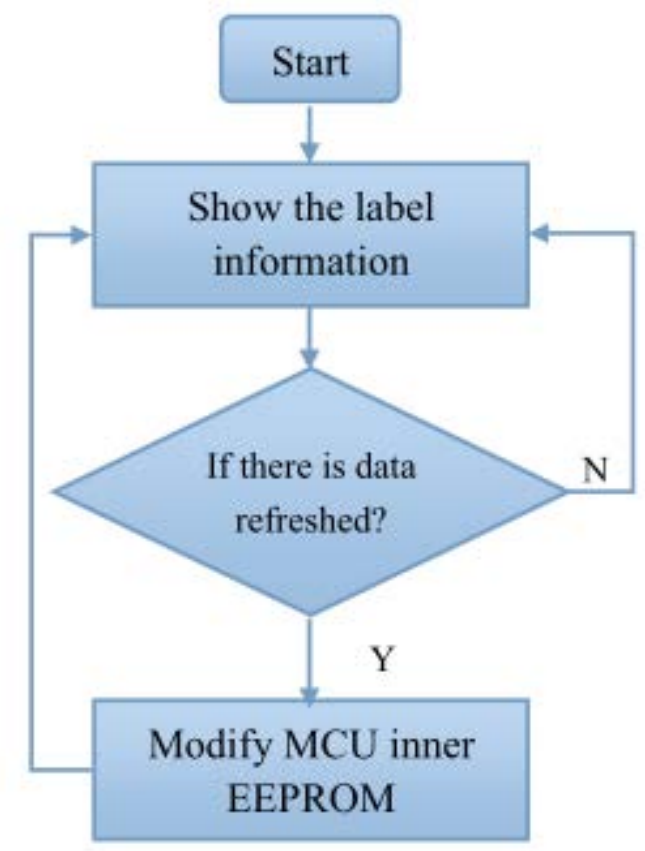

Figure 5: The main program flow chart

\section{Electronic price tag application example}

Designed 50 electronic price tags and a relay consist of test networks. In tests, remote or wireless data can be transferred through the relay to the electronic price tag. During cumulative 2,000 times data modification, there are only two data errors occurring. Among 50 electronic price tags with button batteries powered, the longest working time is 461 days, and the shortest working time is 372 days. From the test, it was found that the working life in general is related to the number of update data and 
the more times electronic price tag is updated, the shorter working time will be. The working hours of electronic price tag is relatively long with tag fewer updated data.

\section{Summary}

This design introduces a low-power wireless electronic price tag from both hardware and software aspects. Designed wireless electronic price tag can receive commodity information wirelessly and have more than one-year life cycle. This design can be used for medium and large retail stores and conducive to the digital management of commodities. The system is low-cost. It is easy to use and promote the system.

\section{References}

[1] Wang Yingzhi, Yang Jia, Han Tailin. Design based on the STM32 RS232-CAN communication protocol converter [J] Manufacturing Automation, 2013,35 (7): 141-143.

[2] Miao Yiru, Hua Ze Xi, Li Guosheng mining secretary-control turnout system based on PLC and CC1100 [J] Instrument Technique and Sensor, 2013,4: 43-46.

[3] Dalian Jin Xian Co.Ltd Electronic Paper Displays data sheet [EB / OL] http: // www good-display.com, 2012. 